\title{
QSOFA and SOFA scores are valuable tools for predicting postoperative sepsis resulting from ureteroscopic lithotripsy (URSL)
}

Chun-Yo Laih

China Medical University Hospital

Po-Jen Hsiao

China Medical University Hospital

Po-Fan Hsieh

China Medical University Hospital

Yu-De Wang

China Medical University Hospital

Chun-Ming Lai

Tunghai University

Chao-Tung Yang

Tunghai University

Chu-Hsing Lin

Tunghai University

Chi-Ping Huang ( D28879@mail.cmuh.org.tw)

China Medical University Hospital

\section{Research Article}

Keywords: SOFA, qSOFA, Ureteroscopy lithotripsy (URSL), Anesthesiologists Classification (ASA), Society of Critical Care Medicine (SCCM)

Posted Date: July 19th, 2021

DOI: https://doi.org/10.21203/rs.3.rs-709356/v1

License: (c) (i) This work is licensed under a Creative Commons Attribution 4.0 International License. Read Full License 


\section{Abstract \\ Purpose}

The SOFA and qSOFA scores are new tools which are used to assess sepsis based on the Third International Consensus Definitions for Sepsis and Septic Shock Task Force. This study aimed to evaluate the feasibility of using the SOFA and qSOFA to predict post-ureteroscopic lithotripsy (URSL) sepsis.

\section{Materials and Methods}

Patients who underwent URSL due to ureteral stone obstruction were retrospectively reviewed using SOFA and qSOFA scores. Patient characteristics including age, gender, comorbidities, American Society of Anesthesiologists Classification (ASA), stone burden, stone location, hydronephrosis status, infectious status, preoperative SOFA and qSOFA score were collected. Preoperative factors were analyzed to determine if they were correlated with postoperative sepsis.

\section{Results}

A total of 830 patients were included in this study, of whom $32(3.9 \%)$ had postoperative sepsis. Multivariate analysis revealed that older age, proximal ureteral stones, severe hydronephrosis, and high preoperative qSOFA or SOFA score were significantly associated with postoperative sepsis. The areas under the curves of a qSOFA score $\geq 1$ and SOFA score $\geq 2$ for predicting postoperative sepsis were 0.754 and 0.823 , respectively.

\section{Conclusions}

Preoperative qSOFA and SOFA scores are convenient and effective for predicting post-URSL sepsis. Further preventive strategies should be performed in these high-risk patients.

\section{Introduction}

Ureteroscopy lithotripsy (URSL) is a commonly used tool for treating ureteral stones.[1] Despite refinement of the instruments and surgical techniques infectious complications still occur, and given that it is an invasive procedure, some morbidities are inevitable. De la Rosette et al. prospectively analyzed 11,885 patients who received URSL and found that the infection rate was $2.8 \%$.[2] The most serious infectious complication, sepsis, is a concern for all physicians because of its high morbidity and mortality.[3] Sepsis is a systemic disease comprising physiological, pathological, and biochemical abnormalities. It used to be defined as a systemic inflammatory response to infection, and it could be diagnosed by meeting two or more Systemic Inflammatory Response Syndrome (SIRS) criteria, including 
tachycardia (heart rate $>90$ beats/min), tachypnea (respiratory rate $>20$ breaths $/ \mathrm{min}$ ), fever or hypothermia (temperature $>38$ or $<36^{\circ} \mathrm{C}$ ), leukocytosis, leukopenia, or bandemia (white blood cells $>$ $1,200 / \mathrm{mm}^{3},<4,000 / \mathrm{mm}^{3}$ or bandemia $\left.\geq 10 \%\right) .[4,5,6]$

How to predict and prevent postoperative sepsis is a very important clinical issue. He et al. reported that the degree of preoperative hydronephrosis was related to postoperative sepsis.[7] Another study reported that postoperative urinary tract infections and urosepsis in URSL were correlated with stone burden.[8] However, the degree of hydronephrosis and stone burden are not currently standardized, and clinical physicians need a more objective and effective tool for predicting sepsis.

In 2016, the Society of Critical Care Medicine (SCCM) and the European Society of Intensive Care Medicine (ESICM) advocated that sepsis should be defined as a life-threatening organ dysfunction caused by a dysregulated host response to infection.[9] The Sequential Organ Failure Assessment (SOFA) score was then proposed as a new scoring system by the SCCM and the ESICM to indicate inflammation or organ dysfunction which could be discovered by bedside examinations and routine laboratory tests. [10] The qSOFA score is a simpler tool that does not require laboratory tests. Changes in the SOFA or qSOFA score $\geq 2$ are associated with a 10- to 12-fold higher mortality rate.[11] Several previous studies have demonstrated that changes in the SOFA or qSOFA score were good predictors of mortality or risk stratification in both intensive care unit (ICU) patients $[9,10,12,13]$ and sepsis patients.[14-16] This study aimed to evaluate the feasibility of using SOFA and qSOFA scores for predicting post-URSL sepsis.

\section{Materials And Methods}

The study protocol was conducted according to the guidelines of the Declaration of Helsinki and approved by the Institutional Review Board of China Medical University Hospital in Taiwan (IRB: CMUH109-REC1-079). Patient consent was waived, due to retrospective study and all data had been deidentified under review by the Institutional Review Board of China Medical University Hospital. A total of 862 patients who underwent URSL between January 1st 2014 and December 31st 2019 at our hospital, a tertiary referral center, due to obstructive ureteral stones were recruited. Thirty-two patients were excluded due to preoperative sepsis as defined by the SIRS criteria. We also excluded patients who had vesical or renal stones to minimize interference. Finally, 830 patients were enrolled in this study.

The URSL procedure was performed using the pneumatic method (Swiss Lithoclast ${ }^{\circledR}$ - Electro Medical Systems) to fragment the stones. Variables of interest, including the patient's gender, age, comorbidities (such as diabetes mellitus (DM) etc.), American Society of Anesthesiologists (ASA) Classification, stone burden, stone location, hydronephrosis status, the pre- and postoperative SIRS score within 24 hours, and the preoperative qSOFA and SOFA scores were recorded.

\section{Definition of SOFA and qSOFA score}


The SOFA score was evaluated using six important systems, including respiration (PaO2/FiO2), coagulation (platelet count), liver (bilirubin), cardiovascular (mean arterial pressure), central nervous system (Glasgow Coma Scale, GCS), and renal system (creatinine and/or urine output).[9] The qSOFA score evaluated systolic blood pressure $(\leq 100 \mathrm{mmHg})$, respiratory rate ( $\geq 22$ breaths per minute) and altered mental status (GCS < 15).[10]

\section{Statistical analysis}

Comparisons between predictive factors and outcomes were assessed using the chi-square test or Fisher's exact test for categorical variables, and the Mann-Whitney test for continuous, normally distributed and skewed variables. One-way analysis of variance (ANOVA) was used to determine differences between the means of two or more independent factors. Multivariate analysis was used to evaluate the possible independent factors associated with postoperative sepsis after adjusting for covariates determined by univariate analysis. Logistic regression was used to determine associations between preoperative qSOFA and SOFA scores and postoperative sepsis. The predictive accuracy of the qSOFA and SOFA scores for postoperative sepsis was evaluated using the area under the curve (AUC) of the receiver operating characteristic (ROC) curve. Cut-off values for the SOFA and qSOFA scores were defined according to Youden's index of ROC curves for postoperative sepsis. A p-value $<0.05$ was considered to indicated a statistically significant difference. All statistical analyses were performed using SPSS (IBM Corp. Released 2013. IBM SPSS Statistics for Windows, Version 22.0).

\section{Results}

A total of 830 patients met the inclusion criteria and underwent URSL during the 72-month study period (Fig. 1), of whom 32 (3.9\%) had sepsis postoperatively. The demographic data and clinical characteristics of those with and without postoperative sepsis are shown in Table 1. 
Table 1

Demographic data and clinical characteristics of the patients who received URSL

\begin{tabular}{|c|c|c|c|}
\hline Characteristic & $\begin{array}{l}\text { Non-postoperative sepsis } \\
(\mathrm{n}=798)\end{array}$ & $\begin{array}{l}\text { Postoperative sepsis } \\
(n=32)\end{array}$ & P-value \\
\hline Mean age \pm SD & $55.4 \pm 12.99$ & $67.38 \pm 11.11$ & $<0.001$ \\
\hline Gender & & & 0.411 \\
\hline Male & $540(67.7 \%)$ & $26(81.3 \%)$ & \\
\hline Female & $258(32.3 \%)$ & $6(18.8 \%)$ & \\
\hline Patient source & & & 0.004 \\
\hline Clinic & $620(77.7 \%)$ & $14(43.8 \%)$ & \\
\hline ER & $178(22.3 \%)$ & $18(56.3 \%)$ & \\
\hline BMI $\left(\mathrm{kg} / \mathrm{m}^{2}\right)$ & $26.23 \pm 4.05$ & $28.34 \pm 2.21$ & 0.002 \\
\hline \multicolumn{4}{|l|}{ Comorbidity } \\
\hline DM & $126(15.8 \%)$ & $14(43.8 \%)$ & 0.009 \\
\hline HTN & $320(40.1 \%)$ & $14(43.8 \%)$ & 0.799 \\
\hline Heart disease & $34(4.3 \%)$ & $4(12.5 \%)$ & 0.163 \\
\hline CKD & $54(6.8 \%)$ & $4(12.5 \%)$ & 0.309 \\
\hline ASA & & & 0.009 \\
\hline ASA I & $156(19.5 \%)$ & $0(0 \%)$ & \\
\hline ASA II & $562(70.4 \%)$ & $22(68.8 \%)$ & \\
\hline ASA III \& IV & $80(10.0 \%)$ & $10(31.3 \%)$ & \\
\hline Preoperative hydronephrosis & & & $<0.001$ \\
\hline Moderate at most & $646(81.0 \%)$ & $10(31.3 \%)$ & \\
\hline Severe & $152(19.0 \%)$ & $22(68.8 \%)$ & \\
\hline Preoperative ESWL* & $78(9.8 \%)$ & $6(18.8 \%)$ & 0.213 \\
\hline Preoperative antibiotics ${ }^{\star \star}$ & $234(29.3 \%)$ & $16(50.0 \%)$ & 0.095 \\
\hline Preoperative PCN & $110(13.8 \%)$ & $6(18.8 \%)$ & 0.478 \\
\hline Stone side & & & 0.494 \\
\hline Unilateral & $766(96.0 \%)$ & $30(93.8 \%)$ & \\
\hline Bilateral & $32(4.0 \%)$ & $2(6.2 \%)$ & \\
\hline
\end{tabular}




\begin{tabular}{|c|c|c|c|}
\hline Characteristic & $\begin{array}{l}\text { Non-postoperative sepsis } \\
(n=798)\end{array}$ & $\begin{array}{l}\text { Postoperative sepsis } \\
(n=32)\end{array}$ & P-value \\
\hline \multicolumn{3}{|l|}{ Stone site } & 0.002 \\
\hline Proximal ureter & $328(41.1 \%)$ & $26(81.3 \%)$ & \\
\hline Mid + distal ureter & $470(58.9 \%)$ & $18.80 \%$ & \\
\hline Mean stone number \pm SD & $1.14 \pm 0.5$ & $1 \pm 0$ & 0.271 \\
\hline Mean stone size $(\mathrm{mm}) \pm \mathrm{SD}$ & $8.26 \pm 3.57$ & $12.75 \pm 3.80$ & $<0.001$ \\
\hline Preoperative qSOFA score & $0.16 \pm 0.44$ & $0.88 \pm 0.81$ & 0.003 \\
\hline Preoperative SOFA score & $0.66 \pm 1.06$ & $2.25 \pm 1.29$ & $<0.001$ \\
\hline \multicolumn{4}{|c|}{ URSL = ureteroscopic lithotripsy } \\
\hline \multicolumn{4}{|l|}{ SD = Standard Deviation } \\
\hline \multicolumn{4}{|l|}{ ER = Emergency Room } \\
\hline \multicolumn{4}{|l|}{ BMI = Body Mass Index } \\
\hline \multicolumn{4}{|l|}{ DM = Diabetes mellitus } \\
\hline \multicolumn{4}{|l|}{ HTN = Hypertension } \\
\hline \multicolumn{4}{|l|}{ CKD $=$ Chronic Kidney Disease } \\
\hline \multicolumn{4}{|c|}{ ASA = American Society of Anesthesiologists classification } \\
\hline \multicolumn{4}{|c|}{ ESWL = Extracorporeal Shock Wave Lithotripsy; } \\
\hline \multicolumn{4}{|c|}{ PCN = Percutaneous Nephrostomy } \\
\hline \multicolumn{4}{|c|}{ (q)SOFA score = (quick) Sequential Organ Failure Assessment score } \\
\hline \multicolumn{4}{|c|}{ * Preoperative ESWL was defined as patients who received ESWL within 30 days preoperatively } \\
\hline
\end{tabular}

The average preoperative SOFA score was 0.72 and the average preoperative qSOFA score was 0.19 (Table 2). The preoperative SOFA and qSOFA scores were significantly higher in the sepsis patients than in the non-sepsis patients (SOFA: 2.25 vs $0.66, p<0.001$; qSOFA: $0.88 \pm 0.81$ vs $0.16 \pm 0.44 ; p=0.003$ ) (Table 1). In addition, the patients were more likely to get postoperative sepsis if they were older $(67.38 \pm$ 11.11 vs $55.4 \pm 12.99$ years; $p<0.001)$, were admitted to the emergency room $(22.3 \%$ vs $56.3 \% ; p=0.004)$, had DM (43.8\% vs $15.8 \% ; p=0.009)$, had a higher ASA (10.0\% vs $31.3 \% ; p=0.009)$, had severe hydronephrosis $(19.0 \%$ vs $68.8 \%$; $p<0.001)$, had proximal ureteral stones $(41.1 \%$ vs $81.3 \% ; p=0.002)$, had larger stones $(12.75 \pm 3.80$ vs $8.26 \pm 3.57 ; p<0.001)$, had a longer operative time $(66.25 \pm 35.58$ vs 51.51 
\pm 25.81 minutes; $p=0.028)$, and a longer hospital stay $(7.25 \pm 2.93$ vs $3.74 \pm 2.09$ days; $p<0.001)$ (Table 1 and Table 3). 
Table 2

Preoperative qSOFA \& SOFA score

\begin{tabular}{|c|c|}
\hline & All patients $(n=830)$ \\
\hline \multicolumn{2}{|l|}{ Preoperative SOFA score: } \\
\hline \multicolumn{2}{|l|}{ Respiration } \\
\hline $\mathrm{PaO} 2 / \mathrm{FiO} 2, \mathrm{~mm} \mathrm{Hg}(\mathrm{kPa}) \pm \mathrm{SD}$ & $485.43 \pm 32.74$ \\
\hline Mean score & $0.02 \pm 0.15$ \\
\hline \multicolumn{2}{|l|}{ Coagulation } \\
\hline Platelets, $\times 10^{3} / \mu \mathrm{L} \pm \mathrm{SD}$ & $23.037 \pm 65.57$ \\
\hline Mean score & $0.11 \pm 0.53$ \\
\hline \multicolumn{2}{|l|}{ * Liver $(n=76)$} \\
\hline Bilirubin, $\mathrm{mg} / \mathrm{dL}(\mu \mathrm{mol} / \mathrm{L}) \pm \mathrm{SD}$ & $0.9 \pm 0.62$ \\
\hline Mean score & $0.32 \pm 0.59$ \\
\hline \multicolumn{2}{|l|}{ Cardiovascular } \\
\hline Mean arterial pressure, $\mathrm{mmHg} \pm \mathrm{SD}$ & $78.93 \pm 5.74$ \\
\hline Mean score & $0.07 \pm 0.26$ \\
\hline \multicolumn{2}{|l|}{ Central nervous system } \\
\hline Glasgow Coma Scale score \pm SD & $14.92 \pm 0.29$ \\
\hline Mean score & $0.07 \pm 0.26$ \\
\hline \multicolumn{2}{|l|}{ Renal } \\
\hline Creatinine, $\mathrm{mg} / \mathrm{dL}(\mu \mathrm{mol} / \mathrm{L}) \pm \mathrm{SD}$ & $1.20 \pm 0.54$ \\
\hline Mean score & $0.41 \pm 0.67$ \\
\hline Total preoperative SOFA score \pm SD & $0.72 \pm 1.11$ \\
\hline Total preoperative qSOFA score \pm SD & $0.19 \pm 0.48$ \\
\hline \multicolumn{2}{|c|}{ * Not every patient had data for bilirubin $(n=76)$} \\
\hline \multicolumn{2}{|c|}{ (q) SOFA score $=$ (quick) Sequential Organ Failure Assessment score } \\
\hline \multicolumn{2}{|l|}{ SD = Standard Deviation } \\
\hline $\mathrm{ER}=$ Emergency room & \\
\hline
\end{tabular}


Table 3

Surgical outcomes of URSL

\begin{tabular}{|c|c|c|c|}
\hline & $\begin{array}{l}\text { Non-postoperative sepsis } \\
(n=798)\end{array}$ & $\begin{array}{l}\text { Postoperative sepsis } \\
(n=32)\end{array}$ & P-value \\
\hline Mean operative time $(\min ) \pm$ SD & $51.51 \pm 25.81$ & $66.25 \pm 35.58$ & 0.028 \\
\hline Ureteral D-J stenting & $718(90 \%)$ & $26(81.3 \%)$ & 0.224 \\
\hline Mean stenting days \pm SD & $12.32 \pm 12.70$ & $11.31 \pm 13.88$ & 0.756 \\
\hline \multicolumn{3}{|l|}{ Type of anesthesia } & \multirow[t]{3}{*}{0.154} \\
\hline GA & $124(15.5 \%)$ & $10(31.3 \%)$ & \\
\hline SA & $674(84.5 \%)$ & $22(68.7 \%)$ & \\
\hline Postoperative SOFA score & $0.44 \pm 0.89$ & $4.69 \pm 4.29$ & $<0.001$ \\
\hline Days of hospital stay \pm SD & $3.74 \pm 2.09$ & $7.25 \pm 2.93$ & $<0.001$ \\
\hline Stone free* & $758(95.0 \%)$ & $28(87.5 \%)$ & 0.205 \\
\hline \multicolumn{4}{|l|}{ URSL = Ureteroscopic Lithotripsy } \\
\hline \multicolumn{4}{|l|}{ SD = Standard Deviation } \\
\hline \multicolumn{4}{|l|}{ D-J = Double-J Ureteral Stenting } \\
\hline \multicolumn{4}{|l|}{$\mathrm{GA}=$ General Anesthesia } \\
\hline \multicolumn{4}{|l|}{ SA = Spinal Anesthesia } \\
\hline SOFA score = Sequential Organ $\mathrm{F}$ & ailure Assessment score & & \\
\hline
\end{tabular}

Multivariate analysis revealed that postoperative sepsis was significantly associated with older age $(\mathrm{p}=$ 0.04; OR:1.119 (95\% Cl 1.036-1.209)), proximal stones ( $p=0.031$; OR: 1.138 (95\% Cl 1.095-2.043)), longer operative time ( $p=0.028$; OR: 1.25 (95\% Cl 1.035-1.671)), and severe hydronephrosis $(p=0.01$; OR: 7.749 (95\% Cl 1.642-36.575)) (Table 4). The multivariate analysis also revealed that the patients who had higher preoperative qSOFA or SOFA scores were significantly more likely to have postoperative sepsis ( $<$ < 0.001; OR: 51.057; 95\% Cl: 2.381-42.267)) (Table 4). For the qSOFA score, $126(15.2 \%)$ patients scored $\geq 1$ point preoperatively, and $26(3.1 \%)$ of these patients had sepsis postoperatively $(p<$ 0.001; OR: 21.046, accuracy: 74.65\%, sensitivity: 62.5\%, specificity: $86.8 \%$ ) (Table 5). For the SOFA score, $58(7.0 \%)$ patients scored $\geq 2$ points preoperatively, and $26(3.13 \%)$ of these patients had sepsis postoperatively ( $<<0.001$; OR: 38.798 , accuracy: $83.6 \%$, sensitivity: $81.3 \%$, specificity: $85.9 \%$ ) (Table 5 ). The AUC of the ROC curve for qSOFA score was $0.754(95 \% \mathrm{Cl}, 0.651-0.856)$, and the AUC for SOFA score was $0.823(95 \% \mathrm{Cl}, 0.729-0.916)$ (Fig. 2). 
Table 4

Multivariate analysis of factors affecting postoperative sepsis

\begin{tabular}{|lll|}
\hline & P-value & OR (95\% Cl) \\
\hline Age & 0.004 & $1.119(1.036-1.209)$ \\
\hline Stone site (proximal ureter or not) & 0.031 & $1.138(1.095-2.043)$ \\
\hline Operative time & 0.028 & $1.25(1.035-1.671)$ \\
\hline Hydronephrosis (severe or not) & 0.01 & $7.749(1.642-36.575)$ \\
\hline * High preoperative qSOFA or & $<0.001$ & $51.057(2.381-42.267)$ \\
\hline SOFA score & & \\
\hline * High score means qSOFA $\geq 1$ or SOFA $\geq 2$ points preoperatively \\
\hline OR = Odds Ratio \\
\hline (q)SOFA score = (quick) Sequential Organ Failure Assessment score \\
\hline
\end{tabular}

Table 5

The association between a high qSOFA or SOFA score* and postoperative sepsis

\begin{tabular}{|c|c|c|c|c|}
\hline & $\begin{array}{l}\text { Non-postoperative sepsis } \\
(n=798)\end{array}$ & $\begin{array}{l}\text { Postoperative sepsis } \\
(n=32)\end{array}$ & P-value & Odds ratio \\
\hline \multicolumn{3}{|l|}{ qSOFA } & \multirow[t]{3}{*}{$<0.001$} & \multirow{3}{*}{$\begin{array}{l}21.046 \\
(4.615-95.987)\end{array}$} \\
\hline qSOFA = 0 & $698(87.5 \%)$ & $6(18.8 \%)$ & & \\
\hline qSOFA $\geq 1$ & $100(12.5 \%)$ & $26(81.3 \%)$ & & \\
\hline \multicolumn{3}{|l|}{ SOFA } & \multirow[t]{3}{*}{$<0.001$} & \multirow{3}{*}{$\begin{array}{l}38.798 \\
(8.204-183.476)\end{array}$} \\
\hline SOFA $<2$ & $766(96.0 \%)$ & $6(18.8 \%)$ & & \\
\hline SOFA $\geq 2$ & $32(4.0 \%)$ & $26(81.2 \%)$ & & \\
\hline \multicolumn{5}{|c|}{ * High qSOFA score means qSOFA $\geq 1$ point, high SOFA score means SOFA $\geq 2$ points } \\
\hline \multicolumn{5}{|c|}{ (q)SOFA score = (quick) Sequential Organ Failure Assessment score } \\
\hline
\end{tabular}

We used one-way ANOVA analysis to determine if the occurrence of comorbidities were associated with age, but these comorbidities were not risk factors for postoperative infection after multivariate analysis (Table 6). Of the 32 patients who developed sepsis, two (6.25\%) had Clavien grade IV complications because of admission to the ICU, four (12.50\%) had Clavien grade III complications because of percutaneous abscess drainage due to perirenal infection, and the others had Clavien grade II complications due to receiving intravenous antibiotics and nutrition. 
Table 6

One-way ANOVA of comorbidities associated with age undergoing sepsis/ non-sepsis

\begin{tabular}{|llll|}
\hline & Age (non-sepsis) & Age (sepsis) & P value \\
\hline DM & $55.10+-13.337$ & $58.92+-10.142$ & $<0.001$ \\
\hline HTN & $51.85+-12.447$ & $61.47+-11.450$ & $<0.001$ \\
\hline CV & $55.17+-12.658$ & $67.22+-13.524$ & $<0.001$ \\
\hline CKD & $56.06+-12.513$ & $51.40+-17.155$ & 0.049 \\
\hline
\end{tabular}

\section{Discussion}

In this study, we aimed to evaluate the ability of SOFA and qSOFA scores to predict postoperative sepsis after URSL. In the multivariate analysis, age, operative time, hydronephrosis, proximal location, SOFA and qSOFA scores were significantly associated with postoperative sepsis. The SOFA and qSOFA scores had the two highest AUCs to predict sepsis. The SOFA and qSOFA scoring systems have different features and are suitable for different situations. Some items on the qSOFA and SOFA are similar, however they are not all the same. The qSOFA score does not require laboratory tests and can be quickly and repeatedly assessed at the bedside, whereas the SOFA score can evaluate the severity of organ dysfunction by quantifying abnormalities using laboratory tests.

All scoring systems, including SOFA, qSOFA and SIRS aim to define sepsis by evaluating the severity of organ dysfunction, however they do this in different ways. Nevertheless, most of these systems emphasize admission to the ICU or emergency department. Seymour et al. compared mortality between patients who were admitted to the ICU and those who were not, and found that SOFA was statistically better at predicting mortality compared with SIRS and qSOFA for this subset of patients.[14] Khwannimit et al. compared hospital and ICU mortality as well as organ failure among qSOFA, SOFA, and SIRS, and they concluded that the SOFA score had significantly better predictive ability.[15] In addition, Fukushima et al. evaluated qSOFA and SOFA scores for predicting mortality in patients with acute pyelonephritis associated with upper urinary tract calculi,[16] and they showed that the SOFA score was a more accurate tool compared with SIRS. However, neither SIRS nor SOFA is intended to be a stand-alone definition of sepsis. At present, SOFA and qSOFA scores are used to assess whether the patient's current physical condition has progressed to severe infection or even septic shock and whether it requires active treatment.

In this study, we wanted to investigate whether the SOFA and qSOFA scores can be used to more accurately evaluate the perfusion function of organs to predict sepsis after surgery. We chose the quantitative SOFA and qSOFA scores because they may more accurately assess the severity of organ dysfunction. We excluded patients with SIRS before surgery. Our results showed that using qSOFA and SOFA scores to predict sepsis or infection-related complications after surgery was more accurate than 
other risk factors, because they do not only represent a single risk factor but an assessment of the entire body system. For example, when considering kidney function alone it is not possible to predict sepsis after surgery,[17] however the qSOFA and SOFA together evaluate multiorgan function and are therefore more predictive than one single factor.

Our results showed that the primary difference between SOFA and qSOFA was that qSOFA used a looser criteria than SOFA. The addition of conditions such as heart, lung, liver and kidney function in SOFA means it will be more accurately predict sepsis. However, we found that all of the patients with a SOFA score $\geq 2$ had a qSOFA score $>1$, suggesting that the qSOFA score can be used to quickly screen patients who may have sepsis after surgery. In clinical practice, if a patient cannot afford to pay for blood tests, preoperative qSOFA scores could be used as a first screening tool. Consequently, patients with a qSOFA score $>1$ should subsequently receive SOFA score evaluation to predict postoperative sepsis more precisely. Therefore, we recommend that patients with a qSOFA score $>1$ should receive further laboratory examinations, including kidney and liver function to screen whether they have potential preoperative organ failure. If the patient has a SOFA score $\geq 2$, they should undergo preoperative preparation, including antibiotic treatment, nutritional support, percutaneous nephrostomy tube insertion for renal pelvis pressure decompression, minimization of the operative time, and monitoring of the pressure inside the renal pelvis during surgery to avoid excessive water pressure perfusion, etc. Taking these steps would help clinicians in the perioperative setting and during postoperative care to prevent postoperative sepsis.

In the current study, the multivariate analysis revealed that age was a significant risk factor for infection after URSL. A previous meta-analysis study also highlighted that elderly patients in some prospective studies had a higher risk of infection.[18] As the elderly are more likely to suffer from DM, cardiovascular disease and chronic obstructive pulmonary disease, sepsis often cannot be controlled once they have infection resistance.[19]

Our results also showed that the patients with more proximal stones were more prone to postoperative infections, although other studies have not found an association between the location of stones and postoperative infections.[20] A possible reason for this difference is that the higher the calculus, the higher the complexity of the operation, which can lead to higher intrarenal pressure and a higher risk of sepsis. During the operation, the renal pelvis must be continuously rinsed to provide the surgeon with an appropriate field of vision.

Southern et al. and Moses et al. reported that excessive operation time was associated with infection after URSL.[21, 22] A longer operation time in our study was associated with postoperative sepsis, and the average operation time in our study was about 66 minutes. The length of the operation may indicate the complexity of the stone location, the patient's anatomy, or the high pressure caused by the amount of fluid installation from the URSL in the renal pelvis during ureteroscopy.[23]

Diabetes is an important risk factor, and diabetic patients are generally believed to have a higher frequency of urinary tract infections,[24] possibly due to an impaired immune system and white blood cell 
function.[25] Although our study did not indicate that DM was a significant predictor in the multivariate analysis, it is still important to carefully consider a patient's history of DM.

The severity of hydronephrosis was significantly associated with postoperative infection in the present study. When hydronephrosis accumulates to a severe degree, the high pressure in the renal pelvis may allow bacteria and endotoxins in the urine to be absorbed into the bloodstream, causing postoperative fever, bacteremia, and even sepsis.[26] However, a limitation of the current study was that that we did not evaluate the pressure in the renal pelvis.

There were some other limitations to this study. First, it was a retrospective study at a single institution and only about $10 \%$ of the patients had their bilirubin level checked preoperatively. The bilirubin level of the patients who were not checked preoperatively was scored zero. Second, all operations were performed by residents and attending doctors with various degrees of experience. Third, we defined preoperative sepsis using the SIRS criteria because the SOFA score states that patients with sepsis must have an acute change $\geq 2$ points following a dynamic review. We could only define sepsis using the SIRS criteria at a single point. Finally, our results cannot be applied to patients receiving retrograde intrarenal surgery, percutaneous nephrolithotomy, or ureterolithotomy.

\section{Conclusions}

The current study demonstrated that preoperative qSOFA and SOFA scores are convenient and effective tools for predicting post-URSL sepsis. Further preventive strategies should be performed in these high-risk patients. However, our results still need further external validation.

\section{Declarations}

\section{Acknowledgements}

N/A

\section{Author Contributions}

PJH and CYL designed the whole work and wrote the manuscript. PFH and YDW helped in analyzing the clinical data and modified the manuscript. CML, CTY and CHL provided designed the statistical methods. $\mathrm{CPH}$ supervised and conducted the whole discussion and final interpretations.

\section{Competing Interests}

None of the authors have any competing interests to declare.

\section{Data Availability}


The datasets generated during and/or analysed during the current study are available from the corresponding author on reasonable request.

\section{References}

1. Türk, C. et al. Guidelines on urolithiasis. The European Association of Urology (EAU)(2017).

2. de la Rosette, J. et al. The clinical research office of the endourological society ureteroscopy global study: indications, complications, and outcomes in 11,885 patients. J Endourol, 28, 131-139 https://doi.org/10.1089/end.2013.0436 (2014).

3. Mariappan, P. \& Tolley, D. A. Endoscopic stone surgery: minimizing the risk of post-operative sepsis. Curr Opin Urol, 15, 101-105 (2005).

4. American College of Chest Physicians/Society of Critical Care Medicine Consensus Conference: definitions for sepsis and organ failure and guidelines for the use of innovative therapies in sepsis. Crit Care Med 20, 864-874 (1992).

5. Bone, R. C. et al. Definitions for sepsis and organ failure and guidelines for the use of innovative therapies in sepsis. The ACCP/SCCM Consensus Conference Committee. American College of Chest Physicians/Society of Critical Care Medicine. Chest 101, 1644-1655, doi:10.1378/chest.101.6.1644 (1992).

6. Marik, P. E., Taeb, A. M. \& SIRS qSOFA and new sepsis definition. J Thorac Dis, 9, 943-945 https://doi.org/10.21037/jtd.2017.03.125 (2017).

7. He, Z., Tang, F., Lei, H., Chen, Y. \& Zeng, G. Risk factors for systemic inflammatory response syndrome after percutaneous nephrolithotomy. Prog Urol, 28, 582-587 https://doi.org/10.1016/j.purol.2018.06.006 (2018).

8. Lai, W. S. \& Assimos, D. Factors Associated With Postoperative Infection After Percutaneous Nephrolithotomy. Rev Urol, 20, 7-11 https://doi.org/10.3909/riu0778 (2018).

9. Khwannimit, B. A comparison of three organ dysfunction scores: MODS, SOFA and LOD for predicting ICU mortality in critically ill patients. J Med Assoc Thai, 90, 1074-1081 (2007).

10. Minne, L., Abu-Hanna, A. \& de Jonge, E. Evaluation of SOFA-based models for predicting mortality in the ICU: A systematic review. Crit Care, 12, R161 https://doi.org/10.1186/cc7160 (2008).

11. Freund, Y. et al. Prognostic Accuracy of Sepsis-3 Criteria for In-Hospital Mortality Among Patients With Suspected Infection Presenting to the Emergency Department. JAMA, 317, 301-308 https://doi.org/10.1001/jama.2016.20329 (2017).

12. Granholm, A., Moller, M. H., Krag, M., Perner, A. \& Hjortrup, P. B. Predictive Performance of the Simplified Acute Physiology Score (SAPS) II and the Initial Sequential Organ Failure Assessment (SOFA) Score in Acutely III Intensive Care Patients: Post-Hoc Analyses of the SUP-ICU Inception Cohort Study. PLoS One, 11, e0168948 https://doi.org/10.1371/journal.pone.0168948 (2016).

13. Safari, S. et al. Accuracy of SOFA score in prediction of 30-day outcome of critically ill patients. Turk J Emerg Med, 16, 146-150 https://doi.org/10.1016/j.tjem.2016.09.005 (2016). 
14. Seymour, C. W. et al. Assessment of Clinical Criteria for Sepsis: For the Third International Consensus Definitions for Sepsis and Septic Shock (Sepsis-3). JAMA, 315, 762-774 https://doi.org/10.1001/jama.2016.0288 (2016).

15. Khwannimit, B., Bhurayanontachai, R. \& Vattanavanit, V. Comparison of the performance of SOFA, qSOFA and SIRS for predicting mortality and organ failure among sepsis patients admitted to the intensive care unit in a middle-income country. J Crit Care, 44, 156-160 https://doi.org/10.1016/j.jcrc.2017.10.023 (2018).

16. Fukushima, H., Kobayashi, M., Kawano, K. \& Morimoto, S. Performance of Quick Sequential (Sepsis Related) and Sequential (Sepsis Related) Organ Failure Assessment to Predict Mortality in Patients with Acute Pyelonephritis Associated with Upper Urinary Tract Calculi. The Journal of urology, 199, 1526-1533 https://doi.org/10.1016/j.juro.2017.12.052 (2018).

17. Fünfstück, R., Ott, U. \& Naber, K. G. The interaction of urinary tract infection and renal insufficiency. Int J Antimicrob Agents, 28 Suppl (1), S72-77 https://doi.org/10.1016/j.ijantimicag.2006.05.004 (2006).

18. Sun, J., Xu, J. \& OuYang, J. Risk Factors of Infectious Complications following Ureteroscopy: A Systematic Review and Meta-Analysis. Urol Int, 104, 113-124 https://doi.org/10.1159/000504326 (2020).

19. Daels, F. P. et al. Age-related prevalence of diabetes mellitus, cardiovascular disease and anticoagulation therapy use in a urolithiasis population and their effect on outcomes: the Clinical Research Office of the Endourological Society Ureteroscopy Global Study. World J Urol, 33, 859-864 https://doi.org/10.1007/s00345-014-1382-6 (2015).

20. Blackmur, J. P. et al. Analysis of Factors' Association with Risk of Postoperative Urosepsis in Patients Undergoing Ureteroscopy for Treatment of Stone Disease. Journal of endourology, 30, 963-969 https://doi.org/10.1089/end.2016.0300 (2016).

21. Southern, J. B. et al. Risk Factors for Postoperative Fever and Systemic Inflammatory Response Syndrome After Ureteroscopy for Stone Disease. Journal of endourology, 33, 516-522 https://doi.org/10.1089/end.2018.0789 (2019).

22. Moses, R. A., Ghali, F. M., Pais, V. M. Jr. \& Hyams, E. S. Unplanned Hospital Return for Infection following Ureteroscopy-Can We Identify Modifiable Risk Factors? The Journal of urology, 195, 931936 https://doi.org/10.1016/j.juro.2015.09.074 (2016).

23. Jung, H. \& Osther, P. J. Intraluminal pressure profiles during flexible ureterorenoscopy. Springerp/us, 4, 373 https://doi.org/10.1186/s40064-015-1114-4 (2015).

24. Hoepelman, A. I., Meiland, R. \& Geerlings, S. E. Pathogenesis and management of bacterial urinary tract infections in adult patients with diabetes mellitus. Int $J$ Antimicrob Agents, 22 Suppl 2, 35-43 https://doi.org/10.1016/s0924-8579(03)00234-6 (2003).

25. Chen, S. L., Jackson, S. L. \& Boyko, E. J. Diabetes mellitus and urinary tract infection: epidemiology, pathogenesis and proposed studies in animal models. The Journal of urology, 182, S51-56 https://doi.org/10.1016/j.juro.2009.07.090 (2009). 
26. Fan, S. et al. Risk factors of infectious complications following flexible ureteroscope with a holmium laser: a retrospective study. Int J Clin Exp Med, 8, 11252-11259 (2015).

\section{Figures}

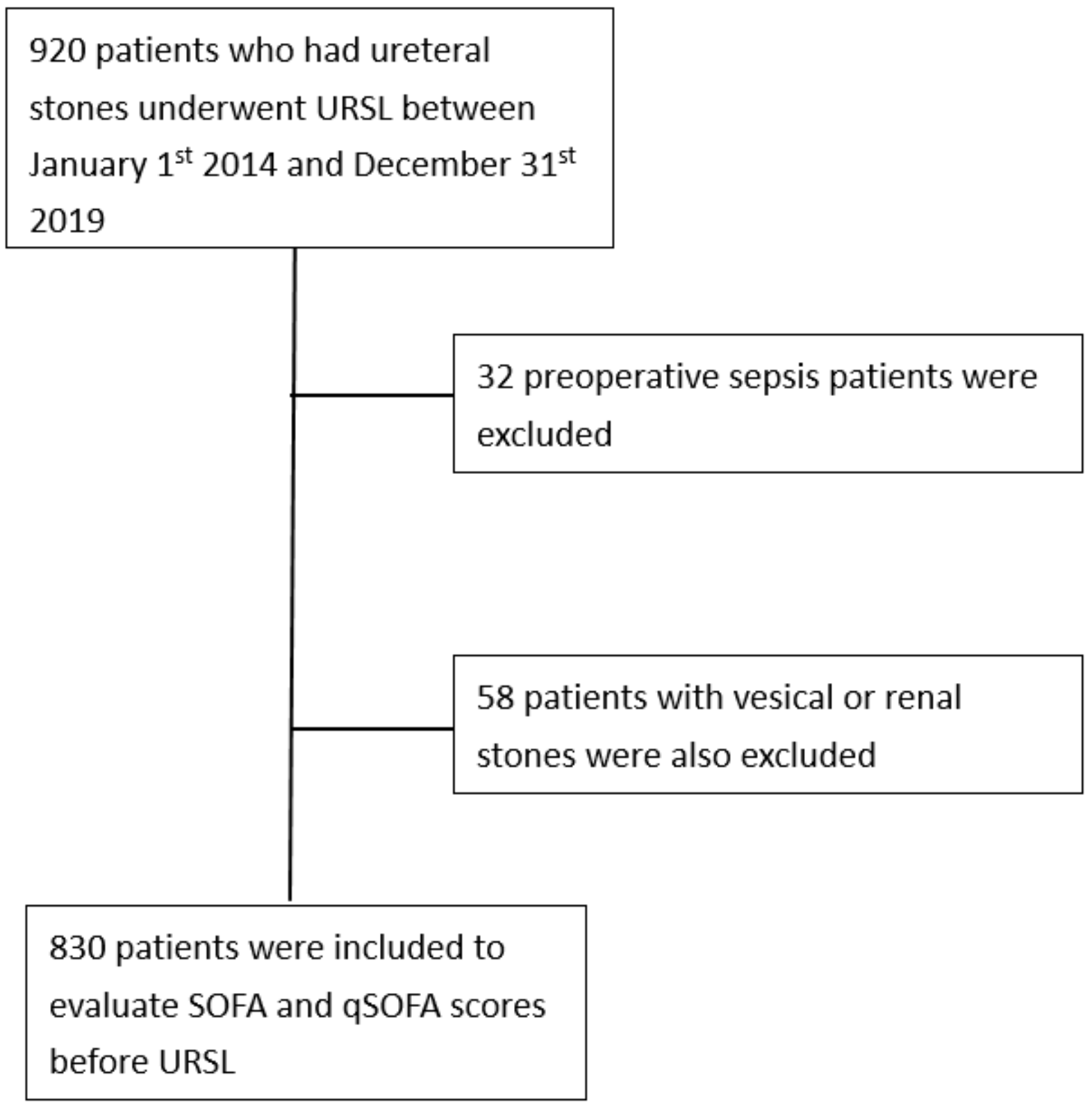

Figure 1

Inclusion and exclusion criteria for the ureteral stone patients in this study Thirty-two patients were excluded because of preoperative sepsis. Besides, 58 patients with vesical or renal stones were also excluded. Total 830 patients were included to the study. 


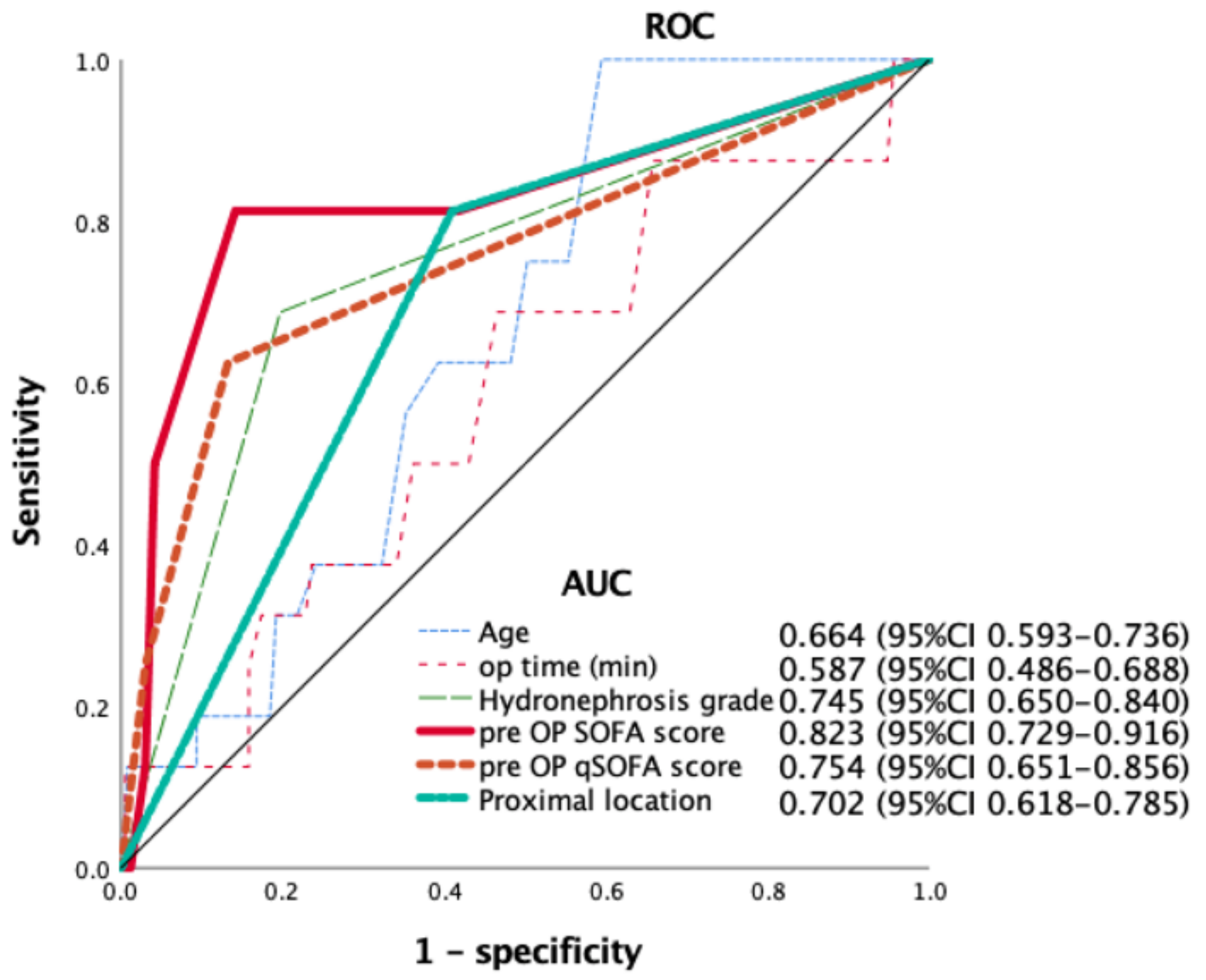

Figure 2

Receiver operating characteristic curves for qSOFA and SOFA to predict sepsis before URSL. Older age, longer operative time, severe hydronephrosis, high preoperative SOFA and qSOFA score, and proximal ureteral stone were significant factors associated postoperative sepsis in multivariate analysis. Besides, the ROC of preoperative SOFA is 0.823 , and 0.754 for preoperative qSOFA, which the most significant factors among them. 